Publicacions Matemàtiques, Vol. 44 (2000), 237-255

\title{
INDICE D'UN HÉRISSON: ÉTUDE ET APPLICATIONS
}

\author{
Yves Martinez-Maure \\ Dédié à Marguerite Borhis
}

\begin{abstract}
Hedgehogs are a natural generalization of convex bodies of class $C_{+}^{2}$. After recalling some basic facts concerning this generalization, we use the notion of index to study differential and integral geometries of hedgehogs.

As applications, we prove a particular case of the Tennis Ball Theorem and a property of normals to a plane convex body of constant width.
\end{abstract}

\section{Introduction}

La théorie des hérissons, dont nous rappellerons les fondements dans cette introduction, prolonge naturellement celle des corps convexes de classe $C_{+}^{2}$ (c'est-à-dire bordés par une hypersurface de classe $C^{2}$ à courbure de Gauss $>0$ ). L'ensemble $\mathcal{K}$ des corps convexes de classe $C_{+}^{2}$ de l'espace euclidien $\mathbb{R}^{n+1}$ ne constitue pas un espace vectoriel. En revanche, les hérissons de $\mathbb{R}^{n+1}$ forment un $\mathbb{R}$-espace vectoriel dans lequel $\mathcal{K}$ peut être envisagé comme un cône convexe. Plus souple et offrant de nouveaux outils conceptuels, le cadre des hérissons est le cadre adéquat pour aborder divers problèmes de la théorie des corps convexes. Les hérissons interviennent ainsi dans l'étude des corps convexes de largeur constante [8], des hypersurfaces admettant un point isocorde [12] ou encore dans l'étude de certaines inégalités géométriques concernant les corps convexes [13]. Le problème de Minkowski, ou problème de la courbure de Gauss prescrite pour les hypersurfaces convexes, se pose naturellement pour les hérissons et présente alors un intérêt particulier: le problème se ramène à l'étude d'une EDP de Monge-Ampère qui change de type sur $\mathbb{S}^{n}$, type d'équation pour lequel il n'existe encore aucun résultat global.

1991 Mathematics Subject Classification. 53A04, 53A05, 52A20, 52A38. 
Les hérissons sont également en rapport avec les surfaces minimales (voir [7], [9] et [15]), les hypersurfaces algébriques de $\mathbb{C}^{n}[\mathbf{5}]$, la théorie des singularités (fronts d'onde, $A$-mersions, singularités lagrangiennes et legendriennes: $[\mathbf{5}],[\mathbf{6}],[\mathbf{1 0}]$ et $[\mathbf{1 1}]$ ) ou encore les modèles du plan projectif dans $\mathbb{R}^{3}$ (une version hérisson de la surface romaine de Steiner est présentée dans [11]).

La notion d'indice joue un rôle important dans l'extension de la théorie des corps convexes de classe $C_{+}^{2}$ aux hérissons. Elle fournit une interprétation géométrique naturelle du volume et diverses informations sur la courbure.

La section 2 présentera les propriétés remarquables de l'indice et les premières conséquences géométriques.

La section 3 sera entièrement consacrée aux applications géométriques. Nous présenterons des propriétés géométriques de diverses classes de hérissons de $\mathbb{R}^{2}$ (aire, sens de la concavité par rapport à l'extérieur, nombre de singularités, etc), mais aussi de $\mathbb{R}^{3}$ (propriétés des régions hyperboliques et elliptiques, contour apparent, effet d'une projection orthogonale sur un plan, etc) et plus généralement de $\mathbb{R}^{n+1}$ (singularités et enveloppe convexe). Nous présenterons également plusieurs applications à d'autres sujets. La dualité projective nous permettra tout d'abord de déduire un cas particulier important du théorème de la balle de tennis d'Arnold [1]. Nous démontrerons ensuite une propriété remarquable des normales à un corps convexe plan de largeur constante et de classe $C_{+}^{2}$ : il existe un point par lequel il passe une infinité de normales ou un ouvert formé de points par lesquels il passe au moins 6 normales. Notre résultat, qui sera plus général, correspond à celui établi par E. Heil dans $\mathbb{R}^{3}$ : un corps convexe de largeur constante de $\mathbb{R}^{3}$ contient un point par lequel il passe une infinité de normales ou un ouvert formé de points par lesquels il passe au moins 10 normales (voir $[\mathbf{3}]$ et [4]).

Nous terminerons notre article par une description plus complète de la structure des hérissons plans. Nous remarquerons que l'addition de Minkowski a sur ceux-ci un effet de convexification. Notons que cette propriété de l'addition de Minkowski a déjà été observée pour les parties compactes de $\mathbb{R}^{n}$ (cf. [16, $\left.\left.\S 3.1\right]\right)$.

Mais commençons par rappeler comment sont définis les hérissons et en quoi ils constituent une extension naturelle des corps convexes de classe $C_{+}^{2}$. Pour tous les résultats concernant les corps convexes de classe $C_{+}^{2}$, nous renvoyons le lecteur au livre de R. Schneider [16].

À tout corps convexe $K \subset \mathbb{R}^{n+1}$ est associé une fonction support $h_{K}$ : $\mathbb{S}^{n} \rightarrow \mathbb{R}, p \longmapsto h_{K}(p)=\max \{\langle m, p\rangle \mid m \in K\}$. Lorsque $K$ est de 
classe $C_{+}^{2}$, cette fonction support $h=h_{K}$ est une fonction de classe $C^{2}$ qui détermine le bord de $K$ comme enveloppe de la famille d'hyperplans d'équation $\langle x, p\rangle=h(p)$. Ce bord $\partial K$ est donc l'hypersurface $\mathcal{H}_{h}$ paramétrée par l'application $x_{h}: \mathbb{S}^{n} \rightarrow \partial K \subset \mathbb{R}^{n+1}, p \longmapsto x_{h}(p)$, où $x_{h}(p)$ est l'unique solution du système

$$
\left\{\begin{array}{l}
\langle x, p\rangle=h(p) \\
\langle x, .\rangle=d h_{p}(.)
\end{array},\right.
$$

à savoir $x_{h}(p)=h(p) p+(\operatorname{grad} h)(p)$. Cette paramétrisation $x_{h}$ n'est autre que la réciproque de l'application de Gauss de $\mathcal{H}_{h}$.

Bien sûr, une fonction $h \in C^{2}\left(\mathbb{S}^{n} ; \mathbb{R}\right)$ n'est pas nécessairement la fonction support d'un corps convexe. Mais, nous pouvons toujours lui associer l'enveloppe de la famille d'hyperplans d'équation $\langle x, p\rangle=h(p)$, c'est-à-dire l'hypersurface $\mathcal{H}_{h}$ paramétrée par $x_{h}$. Les parties régulières de cette hypersurface $\mathcal{H}_{h}$ admettent une orientation transverse pour laquelle le vecteur normal unitaire en $x_{h}(p)$ n'est autre que $p$, si bien que $x_{h}$ peut encore s'interpréter comme la réciproque de l'application de Gauss. L'hypersurface $\mathcal{H}_{h}$ a été appelée le hérisson de fonction support $h$ par R. Langevin, G. Levitt et H. Rosenberg [5].

Lorsque $h$ est la fonction support d'un corps convexe de classe $C_{+}^{2}$, la mesure de volume $n$-dimensionnel de $\mathcal{H}_{h}$ admet le produit des rayons de courbure principaux, noté $R_{h}$ et appelé fonction de courbure de $\mathcal{H}_{h}$, comme densité par rapport à la mesure de Lebesgue sphérique $\sigma$. Le volume $n$-dimensionnel de $\mathcal{H}_{h}$ est donc alors défini comme l'intégrale

$$
v_{n}(h)=\int_{\mathbb{S}^{n}} R_{h}(p) d \sigma(p) .
$$

Si l'on considère $R_{h}(p)$ comme le déterminant de l'application tangente de $x_{h}$ en $p$, cette définition s'étend à un hérisson quelconque. Le volume $n$-dimensionnel de $\mathcal{H}_{h}$ s'interprète alors comme la différence

$$
v_{n}(h)=v_{n}^{+}(h)-v_{n}^{-}(h),
$$

où $v_{n}^{+}(h)$ (resp. $\left.v_{n}^{-}(h)\right)$ désigne le volume des régions à (fonction de) courbure positive (resp. négative). Pour $n \geq 2, v_{n}(h)$ est appelé l'aire (algébrique) de $\mathcal{H}_{h}$.

La notion de volume $(n+1)$-dimensionnel d'un corps convexe de $\mathbb{R}^{n+1}$ s'étend également aux hérissons. Comme pour un corps convexe de classe $C_{+}^{2}$, le volume $(n+1)$-dimensionnel d'un hérisson $\mathcal{H}_{h}$ de $\mathbb{R}^{n+1}$ peut être défini comme l'intégrale

$$
v_{n+1}(h)=\frac{1}{n+1} \int_{\mathbb{S}^{n}} h(p) R_{h}(p) d \sigma(p) .
$$


Géométriquement, ce volume s'interprète comme l'intégrale sur $\mathbb{R}^{n+1}$ $\mathcal{H}_{h}$ de l'indice $i_{h}(x)$, défini comme nombre algébrique d'intersection d'une demi-droite orientée d'origine $x$ avec l'hypersurface $\mathcal{H}_{h}$ munie de son orientation transverse (nombre indépendant de la demi-droite pour un ouvert dense de directions).

Plus généralement, la notion de volume mixte de $n+1$ corps convexes de $\mathbb{R}^{n+1}$ s'étend aux hérissons. Comme pour des corps convexes de classe $C_{+}^{2}$, le volume mixte de $n+1$ hérissons $\mathcal{H}_{h_{1}}, \ldots, \mathcal{H}_{h_{n+1}}$ de $\mathbb{R}^{n+1}$ peut être défini comme l'intégrale

$$
v\left(h_{1}, \ldots, h_{n+1}\right)=\frac{1}{n+1} \int_{\mathbb{S}^{n}} h_{n+1}(p) R_{\left(h_{1}, \ldots, h_{n}\right)}(p) d \sigma(p),
$$

où

$$
R_{\left(h_{1}, \ldots, h_{n}\right)}=\frac{1}{n !} \sum_{k=1}^{n}(-1)^{n+k} \sum_{i_{1}<\cdots<i_{k}} R_{\left(h_{i_{1}}+\cdots+h_{i_{k}}\right)}
$$

est la fonction dite de courbure mixte.

Pour les corps convexes de classe $C_{+}^{2}$, les principales inégalités géométriques résultent d'une inégalité entre volumes mixtes de $n+1$ corps convexes de $\mathbb{R}^{n+1}$, appelée inégalité d'Alexandrov-Fenchel. Cette inégalité, qui s'écrit

$$
v\left(h_{1}, \ldots, h_{n+1}\right)^{2} \geq v\left(h_{1}, h_{1}, h_{3}, \ldots, h_{n+1}\right) v\left(h_{2}, h_{2}, h_{3}, \ldots, h_{n+1}\right),
$$

admet une extension partielle aux hérissons qui fournit toute une série d'inégalités géométriques dont une inégalité de type Brünn-Minkowski pour deux hérissons associés à $n-1$ corps convexes [13].

Venons en maintenant à la notion d'indice d'un point par rapport à un hérisson. Étant donné un hérisson $\mathcal{H}_{h}$ de $\mathbb{R}^{n+1}$, l'indice $i_{h}(x)$ peut être défini pour tout $x$ de $\mathbb{R}^{n+1}-\mathcal{H}_{h}$ comme le degré de l'application

$$
f_{h}^{x}: \mathbb{S}^{n} \rightarrow \mathbb{S}^{n}, p \longmapsto \frac{x_{h}(p)-x}{\left\|x_{h}(p)-x\right\|},
$$

c'est-à-dire par

$$
i_{h}(x)=\frac{1}{\omega_{n}} \int_{\mathbb{S}^{n}} \frac{(h(p)-\langle x, p\rangle) R_{h}(p)}{\left\|x_{h}(p)-x\right\|^{n+1}} d \sigma(p),
$$

où $\omega_{n}$ désigne le volume $n$-dimensionnel de $\mathbb{S}^{n}$. Cet indice ne constitue pas une notion nouvelle, mais un cas particulier de l'indice d'un point par rapport à une hypersurface de $\mathbb{R}^{n+1}$. Toutefois, le fait de se restreindre aux hérissons confère à l'indice des propriétés remarquables. Voici en particulier deux propriétés qui seront établies dans la section 2: 
1) Pour tout hérisson $\mathcal{H}_{h}$ de $\mathbb{R}^{2}$, l'indice $i_{h}$ est donné par

$$
i_{h}(x)=1-\frac{1}{2} n_{h}(x),
$$

où $n_{h}(x)$ désigne le nombre de droites support orientées de $\mathcal{H}_{h}$ qui passent par $x$.

2) Si $\mathcal{H}_{h}$ est un hérisson de $\mathbb{R}^{3}$ et $P \subset \mathbb{R}^{3}$ un plan vectoriel, l'indice du hérisson de $P$ donné par la restriction de $h$ à $\mathbb{S}^{2} \cap P$ est presque partout égal à

$$
\frac{1}{2}\left(n_{h}^{+}(x)-n_{h}^{-}(x)\right),
$$

où $n_{h}^{+}(x)$ (resp. $n_{h}^{-}(x)$ ) désigne le nombre de $p \in \mathbb{S}^{2}$ tels que $R_{h}(p)$ est $>0($ resp. $<0)$ et $x_{h}(p)$ situé sur la normale à $P$ en $x$.

Ces deux propriétés seront à l'origine des principales applications géométriques développées dans les sections 2 et 3 .

\section{2. Étude de l'indice}

Nous commençons notre étude par les hérissons plans. Pour un tel hérisson $\mathcal{H}_{h}$, nous pouvons relier l'indice $i_{h}(x)$ au nombre de droites support passant par $x$ :

Théorème 1. Pour tout hérisson $\mathcal{H}_{h}$ de $\mathbb{R}^{2}$, nous avons:

$$
\forall x \in \mathbb{R}^{2}-\mathcal{H}_{h}, i_{h}(x)=1-\frac{1}{2} n_{h}(x),
$$

où $n_{h}(x)$ désigne le nombre de droites support orientées de $\mathcal{H}_{h}$ qui passent par $x$, i.e. le nombre de zéros de la fonction $h_{x}: \mathbb{S}^{1} \rightarrow \mathbb{R}$, $u \longmapsto h(u)-\langle x, u\rangle$.

Preuve: Comme le hérisson $\mathcal{H}_{h}$ se déduit du hérisson $\mathcal{H}_{h_{x}}$ par la translation de vecteur $x$, nous pouvons supposer que $x$ est l'origine de $\mathbb{R}^{2}$ quitte à remplacer $\mathcal{H}_{h}$ par $\mathcal{H}_{h_{x}}$. Supposons donc que $0_{\mathbb{R}^{2}}=(0,0) \in \mathbb{R}^{2}-\mathcal{H}_{h}$ et considérons $i_{h}\left(0_{\mathbb{R}^{2}}\right)$. Nous obtenons

$$
\begin{aligned}
i_{h}\left(0_{\mathbb{R}^{2}}\right) & =\frac{1}{2 \pi} \int_{0}^{2 \pi} \frac{h(\theta)\left(h+h^{\prime \prime}\right)(\theta)}{h(\theta)^{2}+h^{\prime}(\theta)^{2}} d \theta \\
& =1+\frac{1}{2 \pi} \int_{0}^{2 \pi} \frac{h(\theta) h^{\prime \prime}(\theta)-h^{\prime}(\theta)^{2}}{h(\theta)^{2}+h^{\prime}(\theta)^{2}} d \theta,
\end{aligned}
$$

où $h(\theta)=h(\cos \theta, \sin \theta)$. Autrement dit, nous avons

$$
i_{h}\left(0_{\mathbb{R}^{2}}\right)=1+\frac{1}{2 \pi} \int_{\Gamma_{h}} \omega
$$


où $\Gamma_{h}$ est la courbe de $\mathbb{R}^{2}$ définie par $\gamma_{h}:[0,2 \pi] \rightarrow \mathbb{R}^{2}, \theta \longmapsto\left(h(\theta), h^{\prime}(\theta)\right)$ et $\omega$ la 1-forme différentielle définie par

$$
\omega\left(x_{1}, x_{2}\right)=\frac{x_{1} d x_{2}-x_{2} d x_{1}}{x_{1}^{2}+x_{2}^{2}} \text { pour tout }\left(x_{1}, x_{2}\right) \neq(0,0) .
$$

Or, l'intégrale de $\frac{1}{2 \pi} \omega$ sur $\Gamma_{h}$ est l'indice de l'origine par rapport à $\Gamma_{h}$, c'est-à-dire le nombre algébrique de tours que fait $\Gamma_{h}$ autour de l'origine (nombre positif si $\Gamma_{h}$ tourne dans le sens direct et négatif dans le cas contraire). Comme l'abscisse de $\gamma_{h}(\theta)$ croît (resp. décroît) strictement lorsque l'ordonnée de $\gamma_{h}(\theta)$ est $>0($ resp. $<0)$, ce nombre est égal à l'opposé de $\frac{1}{2} n_{h}\left(0_{\mathbb{R}^{2}}\right)$ et nous avons donc bien

$$
i_{h}\left(0_{\mathbb{R}^{2}}\right)=1-\frac{1}{2} n_{h}\left(0_{\mathbb{R}^{2}}\right) .
$$

Remarque. Pour tout $h \in C^{2}\left(\mathbb{S}^{1} ; \mathbb{R}\right)$, la courbe orientée $\mathcal{H}_{h}$ est paramétrée par $x_{h}: \mathbb{S}^{1} \rightarrow \mathbb{R}^{2}$, mais aussi par $x_{g}: \mathbb{S}^{1} \rightarrow \mathbb{R}^{2}$, où $g(u)=-h(-u)$.

Quitte à remplacer $h$ par $g$, nous pouvons donc supposer dans l'étude de $i_{h}$ que l'intégrale de $h$ sur $\mathbb{S}^{1}$ est $\geq 0$.

Corollaire 1 et définition. Pour tout hérisson $\mathcal{H}_{h}$ de $\mathbb{R}^{2}$, l'ensemble

$$
C_{h}=\left\{x \in \mathbb{R}^{2}-\mathcal{H}_{h} \mid i_{h}(x)=1\right\}
$$

est une partie convexe de $\mathbb{R}^{2}$ que nous appellerons l'intérieur convexe de $\mathcal{H}_{h}$.

Preuve: Supposons sans perte de généralité que l'intégrale de $h$ sur $\mathbb{S}^{1}$ est $\geq 0$. En vertu du Théorème $1, C_{h}$ est l'ensemble des $x \in \mathbb{R}^{2}$ pour lesquels la fonction $h_{x}(u)=h(u)-\langle x, u\rangle$ ne s'annule pas sur $\mathbb{S}^{1}$. Par continuité de $h_{x}$ sur $\mathbb{S}^{1}$, cette condition signifie que la fonction $h_{x}$ reste $>0$ sur $\mathbb{S}^{1}$ (sachant que son intégrale sur $\mathbb{S}^{1}$ est $\geq 0$ puisque égale à celle de $h$ ). L'ensemble $C_{h}$ peut donc s'écrire

$$
C_{h}=\bigcap_{u \in \mathbb{S}^{1}} P_{h}^{-}(u)
$$

où $P_{h}^{-}(u)$ est le demi-plan ouvert d'équation $\langle x, u\rangle<h(u)$. Il est donc convexe comme intersection d'ensembles convexes.

Le volume 2-dimensionnel d'un hérisson $\mathcal{H}_{h}$ de $\mathbb{R}^{2}$ est appelé l'aire (algébrique) de $\mathcal{H}_{h}$. Rappelons qu'il est défini par

$$
v_{2}(h)=\frac{1}{2} \int_{0}^{2 \pi} h(\theta)\left(h+h^{\prime \prime}\right)(\theta) d \theta,
$$


où $h(\theta)=h(\cos \theta, \sin \theta)$, et interprété comme l'intégrale

$$
v_{2}(h)=\int_{\mathbb{R}^{2}-\mathcal{H}_{h}} i_{h}(x) d \lambda(x),
$$

où $\lambda$ désigne la mesure de Lebesgue sur $\mathbb{R}^{2}$. Le volume 1-dimensionnel de $\mathcal{H}_{h}$ est quant à lui appelé la longueur (algébrique) de $\mathcal{H}_{h}$.

Le résultat suivant est une conséquence immédiate du Théorème 1.

Corollaire 2. Tout hérisson de $\mathbb{R}^{2}$ dont deux droites support sont confondues, c'est-à-dire tout hérisson de $\mathbb{R}^{2}$ dont la fonction support vérifie $h(-u)=-h(u)$ pour un point $u$ de $\mathbb{S}^{1}$, est d'intérieur convexe vide et donc d'aire négative.

En particulier, si $\mathcal{H}_{h}$ est un hérisson projectif de $\mathbb{R}^{2}$, i.e. si $h(-u)=$ $-h(u)$ pour tout $u \in \mathbb{S}^{1}$, alors $\mathcal{H}_{h}$ est d'intérieur convexe vide et donc d'aire négative. Dire qu'un hérisson $\mathcal{H}_{h}$ de $\mathbb{R}^{2}$ est projectif signifie qu'il admet exactement une droite support non orientée dans chaque direction.

Plus généralement, si $\mathcal{H}_{h}$ est un hérisson de $\mathbb{R}^{2}$ de largeur moyenne nulle, c'est-à-dire si $h(\theta)=h(\cos \theta, \sin \theta)$ vérifie

$$
\int_{0}^{2 \pi}(h(\theta)+h(\theta+\pi)) d \theta=0,
$$

alors $\mathcal{H}_{h}$ vérifie la condition (et donc la conclusion) du Corollaire 2 (la largeur $l_{h}(\theta)$ de $\mathcal{H}_{h}$ dans la direction de $u(\theta)=(\cos \theta, \sin \theta)$ est définie comme la distance signée entre les droites support de vecteurs normaux unitaires $u(\theta)$ et $u(\theta+\pi)$, c'est-à-dire par $l_{h}(\theta)=h(\theta)+h(\theta+\pi)$ ). Notons que les hérissons de largeur moyenne nulle sont aussi les hérissons de longueur nulle. En effet, $(L)$ équivaut à

$$
\int_{0}^{2 \pi} h(\theta) d \theta=0
$$

dont le premier membre est la longueur (i.e. le volume 1-dimensionnel) de $\mathcal{H}_{h}$.

Nous avions déjà remarqué que les hérissons projectifs, et plus généralement les hérissons de largeur moyenne nulle, sont d'aire négative [13]. Nous en avions alors déduit que l'application

$$
h \longmapsto \sqrt{-v_{2}(h)},
$$

définit une norme associée à un produit scalaire sur l'espace vectoriel de ces hérissons définis à une translation près. 
Considérons à présent un hérisson $\mathcal{H}_{h}$ de $\mathbb{R}^{3}$. Pour tout plan vectoriel $P \subset \mathbb{R}^{3}$, nous nous proposons de relier la (fonction de) courbure de $\mathcal{H}_{h}$ à l'indice du hérisson obtenu en projetant orthogonalement la courbe $x_{h}\left(\mathbb{S}^{2} \cap P\right)$ sur le plan $P$.

Lorsque $\mathcal{H}_{h}$ est convexe, ce hérisson s'interprète comme le contour apparent de $\mathcal{H}_{h}$ vu d'un point à l'infini de la droite vectorielle orthogonale à $P$.

Notations. Pour tout $n \in \mathbb{S}^{2}, P_{n}$ désignera le plan vectoriel orienté de vecteur normal unitaire $n, \mathbb{S}_{n}^{2}$ la demi-sphère unité de $\mathbb{R}^{3}$ définie par $\langle p, n\rangle \geq 0$ et $\pi_{n}$ la projection orthogonale sur $P_{n}$. Par ailleurs, $h_{n}$ désignera la restriction de $h$ au cercle unité de $P_{n}$, c'est-à-dire au grand cercle $\mathbb{S}_{n}^{1}=\mathbb{S}^{2} \cap P_{n}$. Rappelons que le hérisson $\mathcal{H}_{h_{n}} \subset P_{n}$ est la projection orthogonale de $x_{h}\left(\mathbb{S}_{n}^{1}\right)$ sur $P_{n}$ :

$$
\mathcal{H}_{h_{n}}=\pi_{n}\left[x_{h}\left(\mathbb{S}_{n}^{1}\right)\right]
$$

Notre résultat est le suivant:

Théorème 2. Soit $\mathcal{H}_{h}$ un hérisson de $\mathbb{R}^{3}$. Quel que soit $n \in \mathbb{S}^{2}$, pour toute valeur régulière $x$ de l'application $x_{h}^{n}: \mathbb{S}_{n}^{2} \rightarrow P_{n}, p \longmapsto \pi_{n}\left[x_{h}(p)\right]$, nous avons

$$
i_{h_{n}}(x)=\nu_{h}^{n}(x)
$$

où $\nu_{h}^{n}(x)$ est le nombre algébrique d'intersection de la droite orientée passant par $x$ et dirigée par $n$, que nous noterons $D_{x}(n)$, avec la surface $x_{h}\left(\mathbb{S}_{n}^{2}\right)$ munie de son orientation transverse.

Remarques. 1) L'ensemble $\Omega_{n}(h)$ des valeurs régulières de l'application $x_{h}^{n}$ est un ouvert dense de $P_{n}-\mathcal{H}_{h_{n}}$.

2) Le nombre d'intersection $\nu_{h}^{n}(x)$ est donné par

$$
\nu_{h}^{n}(x)=\nu_{h}^{n}(x)^{+}-\nu_{h}^{n}(x)^{-},
$$

où $\nu_{h}^{n}(x)^{+}$(resp. $\nu_{h}^{n}(x)^{-}$) est le nombre de points $p \in \mathbb{S}_{n}^{2}$ tels que $x_{h}(p) \in D_{x}(n)$ et $R_{h}(p)>0$ (resp. $\left.<0\right)$. En effet, l'application tangente

$$
T_{p} x_{h}: T_{p} \mathbb{S}^{2} \rightarrow T_{x_{h}(p)} \mathbb{S}^{2}=T_{p} \mathbb{S}^{2}
$$

conserve ou inverse l'orientation suivant que $R_{h}(p)=\operatorname{dét}\left[T_{p} x_{h}\right]$ est $>0$ ou $<0$.

Preuve: Nous pouvons identifier $P_{n}$ au plan euclidien $\mathbb{R}^{2}$ et supposer sans perte de généralité que $x$ en est l'origine. Pour $x=0_{P_{n}} \in P_{n}-\mathcal{H}_{h_{n}}$, nous obtenons

$$
i_{h_{n}}(x)=\frac{1}{2 \pi} \int_{\mathcal{H}_{h_{n}}} \omega
$$

où $\omega$ est la 1-forme différentielle définie dans la preuve du Théorème 1. 
L'indice $i_{h_{n}}(x)$, qui représente le nombre algébrique de tours que le hérisson $\mathcal{H}_{h_{n}}$ fait autour de $x$ dans le plan orienté $P_{n}$, peut aussi être interprété comme le nombre algébrique de tours que la courbe orientée $x_{h}\left(\mathbb{S}_{n}^{1}\right)$ fait autour de la droite orientée $D_{x}(n)$ dans $\mathbb{R}^{3}$. En d'autres termes, il peut s'écrire

$$
i_{h_{n}}(x)=\frac{1}{2 \pi} \int_{x_{h}\left(\mathbb{S}_{n}^{1}\right)} \omega
$$

ce qu'un calcul élémentaire confirme sans difficulté. Nous avons donc

$$
i_{h_{n}}(x)=\frac{1}{2 \pi} \int_{\partial S} \omega,
$$

où $S$ désigne la surface $x_{h}\left(\mathbb{S}_{n}^{2}\right)$ munie de son orientation transverse.

Comme $x$ est une valeur régulière de $x_{h}^{n}$, il existe dans $P_{n}$ un disque fermé $D$ de centre $x$ dont l'image réciproque par $x_{h}^{n}$ admet une partition de la forme

$$
\left(x_{h}^{n}\right)^{-1}(D)=\bigcup_{k=1}^{N} D_{k},
$$

où $\pi_{n} \circ x_{h}$ est un $C^{1}$-difféomorphisme de $D_{k}$ sur $D$ pour tout $k \in$ $\{1, \ldots, N\}$. L'application de la formule de Stokes à la 1-forme fermée $\frac{1}{2 \pi} \omega$ sur le bord orienté de la surface $S$ privée des points intérieurs à $\pi_{n}^{-1}(D)$ nous donne

$$
\frac{1}{2 \pi} \int_{\partial S} \omega=\sum_{k=1}^{N} \frac{1}{2 \pi} \int_{\partial S_{k}} \omega,
$$

où $S_{k}$ désigne la surface $x_{h}\left(D_{k}\right)$ munie de son orientation transverse. Or, le bord orienté de $S_{k}$ fait un tour et un seul autour de la droite $D_{x}(n)$ et ce tour est compté positivement ou négativement selon que l'orientation de $S_{k}$ correspond à celle de $D_{k}$ ou à son opposé, c'est-à-dire selon que la (fonction de) courbure $R_{h}$ est $>0$ ou $<0$ sur $D_{k}$. Par conséquent, il vient

$$
\frac{1}{2 \pi} \int_{\partial S} \omega=\nu_{h}^{n}(x)^{+}-\nu_{h}^{n}(x)^{-},
$$

c'est-à-dire $i_{h_{n}}(x)=\nu_{h}^{n}(x)$

Pour tout hérisson $\mathcal{H}_{h}$ de $\mathbb{R}^{3}$, l'aire projetée de $\mathcal{H}_{h}$ sur $P_{n}$ (i.e. sur le plan vectoriel orienté de vecteur normal unitaire $n$ ) est définie pour tout $n \in \mathbb{S}^{2}$ comme l'intégrale

$$
v_{2}^{n}(h)=\frac{1}{2} \int_{\mathbb{S}^{2}}|\langle p, n\rangle| R_{h}(p) d \sigma(p) .
$$


L'invariance du volume de $\mathcal{H}_{h}$ par translation nous donne

$$
\int_{\mathbb{S}^{2}}\langle p, n\rangle R_{h}(p) d \sigma(p)=0
$$

et par suite

$$
v_{2}^{n}(h)=\int_{\mathbb{S}_{n}^{2}}\langle p, n\rangle R_{h}(p) d \sigma(p) .
$$

Comme $\left|R_{h}(p)\right| d \sigma(p)$ représente l'élément d'aire (absolue) sur $\mathcal{H}_{h}$, il s'ensuit que l'aire projetée de $\mathcal{H}_{h}$ sur $P_{n}$ peut encore s'écrire

$$
v_{2}^{n}(h)=\int_{\Omega_{n}(h)} \nu_{h}^{n}(x) d \lambda(x),
$$

où $\Omega_{n}(h)$ est l'ensemble des valeurs régulières de la restriction de $\pi_{n} \circ x_{h}$ à $\mathbb{S}_{n}^{2}$.

Lorsque le hérisson $\mathcal{H}_{h}$ est convexe, $v_{2}^{n}(h)$ représente l'aire de sa projection orthogonale sur $P_{n}$. Pour un hérisson quelconque, le Théorème 2 nous donne immédiatement le résultat suivant:

Corollaire 3. Soit $\mathcal{H}_{h}$ un hérisson de $\mathbb{R}^{3}$. Pour tout $n \in \mathbb{S}^{2}$, l'aire projetée de $\mathcal{H}_{h}$ sur le plan $P_{n}$ est égale à l'aire du hérisson $\mathcal{H}_{h_{n}} \subset P_{n}$, autrement dit

$$
v_{2}^{n}(h)=v_{2}\left(h_{n}\right)
$$

Notons que ce résultat peut aussi être établi directement, soit en se ramenant au cas d'un hérisson convexe, soit en appliquant la formule de Stokes.

Un hérisson $\mathcal{H}_{h}$ de $\mathbb{R}^{3}$ est dit projectif lorsque sa fonction support vérifie $h(-p)=-h(p)$ pour tout $p \in \mathbb{S}^{2}$. Géométriquement, cela signifie que chaque plan vectoriel de $\mathbb{R}^{3}$ est parallèle à exactement un plan support non orienté de $\mathcal{H}_{h}$. Dans ce cas, quel que soit $n \in \mathbb{S}^{2}, \mathcal{H}_{h_{n}}$ est un hérisson projectif (et donc d'intérieur convexe vide) et le Théorème 1 nous assure que l'indice $i_{h_{n}}$ est $\leq 0$ sur $P_{n}-\mathcal{H}_{h_{n}}$. Le Théorème 2 nous donne donc le résultat suivant:

Corollaire 4. Soit $\mathcal{H}_{h}$ un hérisson projectif de $\mathbb{R}^{3}$. Toute droite transverse à $\mathcal{H}_{h}$ rencontre une région hyperbolique de $\mathcal{H}_{h}$.

Une propriété remarquable des hérissons projectifs de $\mathbb{R}^{3}$ est qu'ils sont d'aire (algébrique) négative: $v_{2}^{+}(h)$, aire totale des régions elliptiques, est inférieure à $v_{2}^{-}(h)$, aire totale des régions hyperboliques [9]. Nous avons les minorations suivantes de l'aire totale des régions hyperboliques: 
Corollaire 5. Soit $\mathcal{H}_{h}$ un hérisson projectif de $\mathbb{R}^{3}$. Pour tout $n \in \mathbb{S}^{2}$, on $a$

$$
\left|v_{2}\left(h_{n}\right)\right| \leq \frac{1}{2} v_{2}^{-}(h)
$$

Preuve: Le hérisson $\mathcal{H}_{h_{n}}$ étant projectif (et donc d'intérieur convexe vide), nous avons $i_{h_{n}} \leq 0$ et donc

$$
\left|v_{2}\left(h_{n}\right)\right|=-\int_{P_{n}-\mathcal{H}_{h_{n}}} i_{h_{n}}(x) d \lambda_{n}(x),
$$

où $\lambda_{n}$ désigne la mesure de Lebesgue sur $P_{n}$. Le Théorème 2 nous donne donc

$$
\begin{aligned}
\left|v_{2}\left(h_{n}\right)\right| & =-\int_{\Omega_{n}(h)} \nu_{h}^{n}(x) d \lambda_{n}(x) \\
& =\int_{\Omega_{n}(h)}\left(\nu_{h}^{n}(x)^{-}-\nu_{h}^{n}(x)^{+}\right) d \lambda_{n}(x) \\
& \leq \int_{\Omega_{n}(h)} \nu_{h}^{n}(x)^{-} d \lambda_{n}(x) \\
& \leq \frac{1}{2} \int_{\mathbb{S}^{2}} R_{h}^{-}(p) d \sigma(p)=\frac{1}{2} v_{2}^{-}(h)
\end{aligned}
$$

où $R_{h}^{-}(p)=\max \left(0,-R_{h}(p)\right)$.

Nous verrons dans la dernière section d'autres propriétés des hérissons projectifs. Mais rappelons dès à présent ce fameux problème soulevé par R. Langevin, G. Levitt et H. Rosenberg dans [5]:

"Existe-t-il un hérisson projectif de $\mathbb{R}^{3}$ dont les seules singularités sont des arêtes de rebroussement (sans queue d'aronde)?"

Rappelons que les singularités génériques des hérissons de $\mathbb{R}^{3}$ sont des arêtes de rebroussement et des queues d'aronde [5].

\section{Applications géométriques}

L'intérieur et l'extérieur d'un hérisson $\mathcal{H}_{h}$ de $\mathbb{R}^{2}$ sont respectivement définis comme les ensembles

$$
I_{h}=\left\{x \in \mathbb{R}^{2}-\mathcal{H}_{h} \mid i_{h}(x) \neq 0\right\} \quad \text { et } \quad E_{h}=\left\{x \in \mathbb{R}^{2}-\mathcal{H}_{h} \mid i_{h}(x)=0\right\} .
$$


Le Théorème 1 nous apprend que tout hérisson de $\mathbb{R}^{2}$ d'intérieur convexe vide tourne sa concavité vers l'extérieur:

Proposition 1. Soit $\mathcal{H}_{h}$ un hérisson de $\mathbb{R}^{2}$ d'intérieur convexe vide. Si $p$ est un point régulier de $x_{h}: \mathbb{S}^{1} \rightarrow \mathbb{R}^{2}$, alors la droite support d'équation $\langle x, p\rangle=h(p)$ ne rencontre pas l'extérieur de $\mathcal{H}_{h}$ au voisinage de $x_{h}(p)$.

Preuve: Pour tout $q \in \mathbb{S}^{1}$, nous noterons $D_{q}$ la droite support d'équation $\langle x, q\rangle=h(q)$. D'après le Théorème 1 , il suffit de prouver que tout $x \in D_{p}-\left\{x_{h}(p)\right\}$ assez proche de $x_{h}(p)$ vérifie $n_{h}(x)>2$. Rappelons que $n_{h}(x)$ désigne le nombre de droites support orientées de $\mathcal{H}_{h}$ qui contiennent $x$, i.e. le nombre de zéros de la fonction $h_{x}: \mathbb{S}^{1} \rightarrow \mathbb{R}$, $q \longmapsto h(q)-\langle x, q\rangle$. Rappelons également que $h_{x}(q)$ est la distance signée du point $x$ à la droite $D_{q}$.

Supposons sans perte de généralité que $x_{h}(p)$ est l'origine de $\mathbb{R}^{2}$. Quitte à considérer $\mathcal{H}_{h}$ comme le hérisson de fonction support $g(q)=$ $-h(-q)$, nous pouvons également supposer que la (fonction de) courbure est $>0$ en $p$.

Étant donné $p^{\prime} \in \mathbb{S}^{1}-\{p\}$, on a alors: pour tout $x \in D_{p}-\left\{x_{h}(p)\right\}$ assez proche de $x_{h}(p)$, il existe un arc $\Gamma \subset \mathbb{S}^{1}$ issu de $p$ et ne contenant pas $p^{\prime}$ tel que $h_{x}$ est $<0$ aux points intérieurs de $\Gamma$ et change de signe aux extrémités de $\Gamma$. Si $h_{x}\left(p^{\prime}\right) \leq 0$, l'existence de cet arc nous assure que $n_{h}(x)$ est $>2$. Le résultat est donc établi si un $p^{\prime} \in \mathbb{S}^{1}-\{p\}$ est tel que $h\left(p^{\prime}\right)<0$ car alors, tout $x \in D_{p}$ assez proche de $x_{h}(p)=0_{\mathbb{R}^{2}}$ vérifie $h_{x}\left(p^{\prime}\right)<0$. Supposons donc que $h$ est une fonction $\geq 0$.

Quel que soit $q \in \mathbb{S}^{1}, h$ admet alors un zéro sur le demi-cercle (fermé) de $\mathbb{S}^{1}$ qui relie $q$ à son antipodique $-q$ en parcourant $\mathbb{S}^{1}$ dans le sens direct. En effet, on observe dans le cas contraire que l'intérieur convexe de $\mathcal{H}_{h}$ est non vide: on choisit un $\varepsilon \in \mathbb{R}_{+}^{*}$ strictement inférieur à la plus petite valeur prise par $h$ sur ce demi-cercle, on considère le point $q_{\perp} \in \mathbb{S}^{1}$ tel que $\left(q, q_{\perp}\right)$ est une base orthonormale directe de $\mathbb{R}^{2}$ et on vérifie que $\varepsilon q_{\perp}$ est dans l'intérieur convexe de $\mathcal{H}_{h}$.

Or, si $h$ est $>0$ en $-p$ et sur une des deux composantes de $\mathbb{S}^{1}-\{-p, p\}$, alors $h$ est $>0$ sur tout un demi-cercle (fermé) de $\mathbb{S}^{1}$. Par conséquent, seuls les deux cas suivants restent possibles: (1) les deux droites support $D_{p}$ et $D_{-p}$ sont confondues; (2) les deux composantes de $\mathbb{S}^{1}-\{-p, p\}$ contiennent un zéro de $h$. Dans le cas (1), le résultat découle du fait que $h_{x}(-p)=0$ pour tout $x \in D_{p}$. Dans le cas (2), il découle du fait que, pour tout $x \in D_{p}-\left\{x_{h}(p)\right\}$, la fonction $h_{x}$ doit être $<0$ en l'un des zéros de $h$ sur $\mathbb{S}^{1}-\{p\}$. 
Le résultat suivant en découle immédiatement:

Proposition 2. Soit $\mathcal{H}_{h}$ un hérisson de $\mathbb{R}^{2}$. Si $\mathcal{H}_{h}$ est d'intérieur convexe vide (par exemple projectif ou de largeur moyenne nulle), alors $\mathcal{H}_{h}$ est dans l'enveloppe convexe de ses singularités et $x_{h}: \mathbb{S}^{1} \rightarrow \mathbb{R}^{2}$ admet au moins 4 points singuliers.

Un hérisson $\mathcal{H}_{h}$ de $\mathbb{R}^{n+1}$ est dit projectif si $h(-p)=-h(p)$ pour tout $p \in \mathbb{S}^{n}$. Cela signifie qu'il a exactement un hyperplan support (non orienté) parallèle à un hyperplan donné. Les hérissons projectifs sont donc des hypersurfaces singulières. Leurs singularités sont telles que:

Théorème 3. Tout hérisson projectif de $\mathbb{R}^{n+1}$ est dans l'enveloppe convexe de ses singularités.

Ce théorème se déduit de la Proposition 2 par une récurrence sur la dimension de l'espace qui repose sur le lemme suivant:

Lemme et définition. Soit $\mathcal{H}_{h}$ un hérisson de $\mathbb{R}^{n+1}$, où $n \geq 2$. Pour tout hyperplan vectoriel $H \subset \mathbb{R}^{n+1}$, la restriction de $h$ à la sphère unité de $H$ définit un hérisson de $H$ dont la réalisation géométrique est la projection orthogonale de $x_{h}\left[\mathbb{S}^{n} \cap H\right]$ sur $H$. Ce hérisson de $H$ est appelé le hérisson de projection de $\mathcal{H}_{h}$ sur $H$.

La preuve du Théorème 3 , dont nous omettons quelques détails tant elle est semblable à celle d'un cas particulier exposé dans $[\mathbf{1 1}$, th. 4 , p. 595], se présente alors comme suit:

Nous savons que le résultat est vérifié dans $\mathbb{R}^{2}$ en vertu de la Proposition 2. Nous supposons qu'il est vérifié dans $\mathbb{R}^{n}(n \geq 2)$. Nous considérons un hérisson projectif $\mathcal{H}_{h}$ de $\mathbb{R}^{n+1}$ dont nous supposons qu'il n'est pas dans l'enveloppe convexe de ses singularités. Cela signifie que l'enveloppe convexe de $\mathcal{H}_{h}$ a un hyperplan support ne portant aucune singularité de $\mathcal{H}_{h}$. Pour tout hyperplan vectoriel $H$ perpendiculaire à un tel hyperplan, il apparaît alors que le hérisson de projection de $\mathcal{H}_{h}$ sur $H$ peut être considéré comme un hérisson projectif de $\mathbb{R}^{n}$ qui n'est pas dans l'enveloppe convexe de ses singularités, ce qui contredit l'hypothèse de récurrence.

Pour un hérisson projectif de $\mathbb{R}^{3}$, la même approche (projection orthogonale sur un plan vectoriel et utilisation de la Proposition 2) nous donne immédiatement un résultat plus précis: 
Théorème 4. Soit $\mathcal{H}_{h}$ un hérisson projectif de $\mathbb{R}^{3}$. Vu d'un point à l'infini, le contour apparent de $\mathcal{H}_{h}$ ne présente aucun point elliptique (i.e. aucun point régulier où la courbure de Gauss est $>0$ ).

Remarque. Nous pouvons aussi établir que tout hérisson $\mathcal{H}_{h}$ de $\mathbb{R}^{3}$ de largeur moyenne nulle, i.e. vérifiant

$$
\int_{\mathbb{S}^{2}} h(q) d \sigma(q)=0,
$$

est dans l'enveloppe convexe de ses singularités. La preuve consiste en un raisonnement par l'absurde utilisant des projections orthogonales et le Théorème 1.

La Proposition 2 fournit par dualité projective un cas particulier du théorème de la balle de tennis d'Arnold [1]:

Théorème 5. Soit $\mathcal{C}$ une courbe lisse de $\mathbb{S}^{2}$ qui est fermée, simple et partout transverse aux méridiens. SiC admet au plus 3 points d'inflexion, alors $\mathcal{C}$ est incluse dans un hémisphère ouvert de $\mathbb{S}^{2}$. En particulier, si $\mathcal{C}$ divise $\mathbb{S}^{2}$ en deux parties d'aires égales, alors $\mathcal{C}$ admet au moins 4 points d'inflexion.

N.B.: Un point d'inflexion désigne ici un zéro de la courbure géodésique.

Preuve: En vertu des hypothèses, la courbe $\mathcal{C}$ admet une paramétrisation de la forme suivante:

$$
\begin{aligned}
\gamma_{h}: \mathbb{S}^{1} & \longrightarrow \mathbb{S}^{2} \subset \mathbb{R}^{2} \times \mathbb{R} \\
u & \longmapsto \frac{1}{\sqrt{1+h(u)^{2}}}(u, h(u)),
\end{aligned}
$$

où $h: \mathbb{S}^{1} \rightarrow \mathbb{R}$ est la fonction support d'un hérisson de $\mathbb{R}^{2}$. Les courbes $\mathcal{C}$ et $\mathcal{H}_{h}$ peuvent être vues comme deux courbes projectivement duales: la section de $\mathbb{S}^{2}$ (resp. de $\mathbb{R}^{2} \times\{-1\}$ ) par le plan vectoriel orthogonal à $\left(x_{h}(u),-1\right)\left(\right.$ resp. $\left.\gamma_{h}(u)\right)$ est le grand cercle de $\mathbb{S}^{2}$ tangent à $\mathcal{C}$ en $\gamma_{h}(u)$ (resp. la droite support du hérisson $\mathcal{H}_{h} \times\{-1\} \subset \mathbb{R}^{2} \times\{-1\}$ de vecteur normal $(u,-1))$. Les points d'inflexion de $\mathcal{C}$ correspondent donc exactement aux points singuliers de $x_{h}: \mathbb{S}^{1} \rightarrow \mathbb{R}^{2}$, ce que confirme le calcul donnant la courbure géodésique de $\mathcal{C}$ :

$$
K_{g}=\left(\frac{1+h^{2}}{1+\left\|x_{h}\right\|^{2}}\right)^{\frac{3}{2}} R_{h} .
$$


Supposons que la courbe $\mathcal{C}$ a au plus 3 points d'inflexion. Dans ce cas, l'application $x_{h}: \mathbb{S}^{1} \rightarrow \mathbb{R}^{2}$ a au plus 3 points singuliers et la Proposition 2 nous assure que l'intérieur convexe de $\mathcal{H}_{h}$ est non vide. Considérons un point $x$ de cet intérieur convexe. D'après le Théorème 1 , aucune droite support de $\mathcal{H}_{h}$ ne passe par $x$. Il s'ensuit que la section de $\mathbb{S}^{2}$ par le plan vectoriel orthogonal à $(x,-1)$ est un grand cercle de $\mathbb{S}^{2}$ ne rencontrant pas $\mathcal{C}$. En effet, si ce grand cercle contenait un point de la forme $\gamma_{h}(u)$, alors $(x,-1)$ appartiendrait à la droite support de $\mathcal{H}_{h} \times\{-1\}$ section de $\mathbb{R}^{2} \times\{-1\}$ par le plan vectoriel orthogonal à $\gamma_{h}(u)$. La courbe $\mathcal{C}$ est donc incluse dans l'un des deux hémisphères ouverts bordés par ce grand cercle.

Dans [10], nous avons prouvé de même que la courbe $\mathcal{C}$ admet au moins 6 points d'inflexion si elle est en outre invariante par antipodie (ce dernier résultat peut être vu comme un corollaire du théorème de Möbius sur les courbes projectives planes).

Le Théorème 1 nous fournit aussi un résultat concernant les corps convexes plans de largeur constante:

Théorème 6. Soit $\mathcal{H}_{h}$ un hérisson de largeur constante de $\mathbb{R}^{2}$. Il existe un point de $\mathbb{R}^{2}$ par lequel il passe une infinité de normales à $\mathcal{H}_{h}$ ou un ouvert de $\mathbb{R}^{2}$ formé de points par lesquels il passe au moins 6 normales à $\mathcal{H}_{h}$.

N.B.: Nous considérons tout $u \in \mathbb{S}^{1}$ comme un vecteur normal unitaire à $\mathcal{H}_{h}$ et par conséquent, la droite orientée passant par $x_{h}(u)$ et dirigée par u comme une normale à $\mathcal{H}_{h}$.

Notons que E. Heil a établi un résultat analogue dans $\mathbb{R}^{3}$ : un corps convexe de largeur constante de $\mathbb{R}^{3}$ contient un point par lequel il passe une infinité de normales ou un ouvert formé de points par lesquels il passe au moins 10 normales (voir [3] et [4]). Pour de nombreux autres résultats concernant les corps convexes de largeur constante, nous renvoyons le lecteur au tour d'horizon de G. D. Chakerian et H. Groemer [2].

Preuve: Supposons $\mathcal{H}_{h} \subset \mathbb{R}^{2}$ de largeur constante. Nous avons donc

$$
h=g+r,
$$

où $r=\frac{1}{2}(h(u)+h(-u))$ est une constante et $g(u)=\frac{1}{2}(h(u)-h(-u))$ la fonction support d'un hérisson projectif. Notons que les hérissons $\mathcal{H}_{g}$ et $\mathcal{H}_{h}$ ont exactement les mêmes (droites) normales, si bien qu'il suffit de prouver le théorème pour le hérisson projectif $\mathcal{H}_{g}$. 
Comme $\mathcal{H}_{g}$ est projectif, nous savons qu'il est, soit réduit à un point $x$ de $\mathbb{R}^{2}$, soit d'aire algébrique $<0$ (cf. [8]). Dans le premier cas, notre résultat est vérifié puisque alors toutes les normales de $\mathcal{H}_{g}$ passent par le point $x$. Dans le second cas, il existe un ouvert $\mathcal{U}$ de $\mathbb{R}^{2}-\mathcal{H}_{g}$ sur lequel l'indice $i_{g}$ est $\leq-2$ (sachant que pour un hérisson projectif l'indice est naturellement à valeurs dans $2 \mathbb{Z}$ ). Nous en déduisons par le Théorème 1 que le nombre de zéros de l'application $g_{x}: \mathbb{S}^{1} \rightarrow \mathbb{R}, u \longmapsto g(u)-\langle x, u\rangle$ est $\geq 6$ pour tout $x \in \mathcal{U}$. Comme

$$
\begin{aligned}
g_{x}^{\prime}: \quad \mathbb{S}^{1} \subset \mathbb{R}^{2} & \longrightarrow \mathbb{R} \\
(\cos \theta, \sin \theta) & \longmapsto \frac{d}{d \theta}\left[g_{x}(\cos \theta, \sin \theta)\right],
\end{aligned}
$$

admet au moins autant de zéros que $g_{x}$ et que ses zéros correspondent exactement aux normales de $\mathcal{H}_{g}$ passant par $x$, il en découle qu'il passe au moins 6 normales par tout point de l'ouvert $\mathcal{U}$.

Remarque. Dans ce théorème, la valeur 6 est bien la meilleure possible comme le prouve l'exemple du hérisson projectif défini par $h(\theta)=$ $\sin (3 \theta)$.

Pour finir, présentons une brève description de la structure des hérissons plans. Rappelons que deux hérissons $\mathcal{H}_{g}$ et $\mathcal{H}_{h}$ de $\mathbb{R}^{2}$ définissent la même courbe orientée si leurs fonctions support $g$ et $h$ vérifient:

$$
\forall u \in \mathbb{S}^{1}, g(u)=-h(-u) .
$$

Dans ce cas, seule l'orientation des normales (définie comme dans l'énoncé du Théorème 6) permet de distinguer les deux hérissons. Pour les questions qui ne font pas intervenir cette orientation, nous pouvons donc supposer que $\mathcal{H}_{h}$ est de largeur moyenne $\geq 0$ (i.e. que l'intégrale de $h$ sur $\mathbb{S}^{1}$ est $\geq 0$ ) quitte à remplacer $h$ par $g$.

Restreignons donc notre étude à l'ensemble des hérissons de $\mathbb{R}^{2}$ de largeur moyenne $\geq 0$ et posons

$$
H=\left\{h \in C^{2}\left(\mathbb{S}^{1} ; \mathbb{R}\right) \mid \int_{\mathbb{S}^{1}} h(u) d \sigma(u) \geq 0\right\} .
$$

Pour tout $h \in H$, l'ensemble

$$
K_{h}=\left\{x \in \mathbb{R}^{2} \mid \forall u \in \mathbb{S}^{1},\langle x, u\rangle \leq h(u)\right\},
$$

est une partie convexe de $\mathbb{R}^{2}$ que nous appellerons le corps convexe de $\mathcal{H}_{h}$.

La preuve de la proposition suivante ne présente aucune difficulté particulière (la propriété (v) résulte de la propriété (iv) et de l'inégalité de Brünn-Minkowski): 
Proposition 3. Les propriétés suivantes sont vérifiées:

(i) $\forall h \in H, C_{h}=\stackrel{\circ}{K}_{h}$;

(ii) $K=\left\{h \in H \mid K_{h} \neq \varnothing\right\}$ est un cône convexe de $C^{2}\left(\mathbb{S}^{1} ; \mathbb{R}\right)$;

(iii) Pour tout $h \in K$, la fonction support de $K_{h}$ est de classe $C^{1}$ sur $\mathbb{S}^{1}$

(iv) $\forall\left(h_{1}, h_{2}\right) \in K^{2}, K_{h_{1}}+K_{h_{2}} \subset K_{h_{1}+h_{2}}$ et cette inclusion peut $\hat{e t r e}$ stricte;

(v) $\forall\left(h_{1}, h_{2}\right) \in K^{2}, \sqrt{\operatorname{aire}\left(K_{h_{1}+h_{2}}\right)} \geq \sqrt{\operatorname{aire}\left(K_{h_{1}}\right)}+\sqrt{\operatorname{aire}\left(K_{h_{2}}\right)}$.

Remarques. 1) La propriété (iv) nous dit que l'addition de Minkowski a un effet de convexification sur les hérissons plans, effet déjà observé pour les parties compactes (cf. [16, §3.1]).

2) Nous pouvons étendre légèrement la notion de hérisson plan en prenant comme fonctions support toutes les fonctions de classe $C^{1}$ qui sont des différences de fonctions support de corps convexes plans. Nous parlons alors de hérissons généralisés. L'aire d'un tel hérisson $\mathcal{H}_{h}$ (resp. l'aire mixte de deux tels hérissons $\mathcal{H}_{k}$ et $\mathcal{H}_{l}$ ) peut alors être définie par

$$
\begin{aligned}
v_{2}(h) & =\frac{1}{2} \int_{0}^{2 \pi}\left(h^{2}-\left(h^{\prime}\right)^{2}\right)(\theta) d \theta \\
\text { resp. } v_{2}(k, l) & \left.=\frac{1}{2} \int_{0}^{2 \pi}\left(k l-k^{\prime} l^{\prime}\right)(\theta) d \theta\right) .
\end{aligned}
$$

Pour tout $h \in K$, la propriété (iii) nous permet de décomposer $\mathcal{H}_{h}$ en une somme de hérissons généralisés:

$$
\mathcal{H}_{h}=\mathcal{H}_{k}+\mathcal{H}_{l}
$$

où $k$ est la fonction support de $K_{h}$ et $l$ la différence $h-k$. Nous pouvons alors vérifier que le hérisson généralisé $\mathcal{H}_{l}$ est d'indice $\leq 0$ et vérifie

$$
v_{2}(k, l)=0 \quad \text { et donc } \quad v_{2}(k+l)=v_{2}(k)+v_{2}(l),
$$

de sorte que

$$
v_{2}(k)=v_{2}^{+}(h) \quad \text { et } \quad v_{2}(l)=-v_{2}^{-}(h) .
$$

Pour plus d'informations sur les hérissons généralisés, nous renvoyons le lecteur à $[\mathbf{1 4}]$. 


\section{Références}

[1] V. I. ARnold, "Topological invariants of plane curves and caustics", University Lecture Series 5, American Mathematical Society, Providence, RI, 1994.

[2] G. D. Chakerian et H. Groemer, Convex bodies of constant width, in: "Convexity and its applications", Birkhäuser, BaselBoston, Mass., 1983, pp. 49-96.

[3] E. HeIL, Existenz eines 6-Normalenpunktes in einem konvexen Körper, Arch. Math. (Basel) 32(4) (1979), 412-416.

[4] E. HeIL, Korrectur zu: "Existenz eines 6-Normalenpunktes in einem konvexen Körper", [Arch. Math. (Basel) 32(4) (1979), 412416], Arch. Math. (Basel) 33(5) (1979-80), 496.

[5] R. Langevin, G. Levitt et H. Rosenberg, Hérissons et multihérissons (enveloppes paramétrées par leur application de Gauss), in: "Singularities (Warsaw, 1985)", Banach Center Publ. 20, PWN, Warsaw, 1988, pp. 245-253.

[6] R. Langevin, G. Levitt et H. Rosenberg, Classes d'homotopie de surfaces avec rebroussements et queues d'aronde dans $\mathbb{R}^{3}$, Canad. J. Math. 47(3) (1995), 544-572.

[7] R. Langevin et H. Rosenberg, A maximum principle at infinity for minimal surfaces and applications, Duke Math. J. 57(3) (1988), 819-828.

[8] Y. Martinez-Maure, Hérissons projectifs et corps convexes de largeur constante, C. R. Acad. Sci. Paris Sér. I Math. 321(4) (1995), 439-442.

[9] Y. Martinez-Maure, Hedgehogs and area of order 2, Arch. Math. (Basel) 67(2) (1996), 156-163.

[10] Y. Martinez-Maure, A note on the tennis ball theorem, Amer. Math. Monthly 103(4) (1996), 338-340.

[11] Y. Martinez-Maure, Sur les hérissons projectifs (enveloppes paramétrées par leur application de Gauss), Bull. Sci. Math. 121(8) (1997), 585-601.

[12] Y. Martinez-Maure, Hedgehogs of constant width and equichordal points, Ann. Polon. Math. 67(3) (1997), 285-288.

[13] Y. Martinez-Maure, De nouvelles inégalités géométriques pour les hérissons, Arch. Math. (Basel) 72(6) (1999), 444-453.

[14] Y. Martinez-Maure, Étude des différences de corps convexes plans, Ann. Polon. Math. 72 (1999), 71-78.

[15] H. Rosenberg et E. Toubiana, Complete minimal surfaces and minimal herissons, J. Differential Geom. 28(1) (1988), 115-132. 
[16] R. Schneider, "Convex bodies: The Brünn-Minkowski Theory", Encyclopedia of Mathematics and its Applications 44, Cambridge University Press, Cambridge, 1993.

École Supérieure d'Informatique-Électronique-Automatique

72 , avenue Maurice Thorez

F-94200 Ivry-sur-Seine

France

E-mail address: martinez@esiea.fr

Primera versió rebuda el 23 d'abril de 1999, darrera versió rebuda el 24 de gener de 2000. 